\title{
Effective Methods of Restoring Motion Blurred Images at Arbitrary Direction
}

\author{
Yin Jiang-Yan \\ Jilin Technology College of Electronic Information, \\ Jilin City, China \\ yjy197248@163.com
}

\begin{abstract}
Recovery of degraded images due to motion blurring is a challenging problem in digital imaging. This paper presents a new method of Dirac Delta function algorithm to restore motion blurred images based on an analysis of the characteristics of the inverse filter method and the traditional algebraic method. Experimental results show that the new method is effective in performing motion blurred images at arbitrary direction restoration and extensively validated with different amount of noise in the images.
\end{abstract}

Keywords-Motion blurred images; Dirac Delta function; Inverse filter

\section{INTRODUCTION}

Motion blurring caused by camera shake has been one of the primes causes of poor image quality in digital imaging, especially when using telephoto lenses or long shuttle speeds. In many imaging applications, there is simply not enough light to produce a clear image by using a short shutter speed. As a result, the image will appear blurry due to the relative motion between the camera and the scene. Motion blurring can significantly degrade the visual quality of images. Thus, how to restore motion blurred images has long been a fundamental problem in digital imaging.

Many methods have been proposed to reduce the motion blurring. Motivated greatly by the above-mentioned excellent works, in this paper we will consider a new method to restore motion blurred images based on the good properties of Dirac Delta function. Discrete Dirac Delta function transformed by the same blur parameters in the motion blurred images is used as a basis to restore primely the motion blurred images. This method is still validated different amount of noise in the images.

This paper is organized in three sections including the introduction. The main result is provided in Section 2. Based on mathematical preliminaries, an analysis and implementation progress are described. In Section 3, some meaningful experiments are presented to verify the theories analysis.

\section{ALGORITHM ANALYSIS}

\section{A. Mathematical preliminaries}

Definition 2.1. Dirac Delta function $(\delta)$ :

$$
\begin{aligned}
& \delta(x-c)=0, \quad x \neq c \\
& \int_{a}^{b} \delta(x-c) d x=1, \quad a<c<b
\end{aligned}
$$

where $C$ is a constant.

Property 2.1. Two properties of Dirac Delta function are used in this paper:

1. $\delta(-x)=\delta(x)$

2. $\int_{a}^{b} f(x) \delta(x-c) d x=f(c), \quad a<c<b$

Definition 2.2. The convolution of function $f(x, y)$ and $g(x, y)$ is defined by

$$
\begin{aligned}
& (f * g)(x, y)=\int_{-\infty}^{+\infty} \int_{-\infty}^{+\infty} f(u, v) g(x-u, y-v) d u d v \\
& \text { Property } 2.2 . \\
& (f * g)^{\wedge}=\widehat{f} \cdot \hat{g}
\end{aligned}
$$

\section{B. Implementation}

Suppose $f(x, y)$ is a function obtained from the periodic extension of the original image. By the properties of Dirac Delta function, we have

$$
\begin{aligned}
& f(x, y)=\int_{-\infty}^{+\infty} \int_{-\infty}^{+\infty} f(u, v) \delta(u-x, v-y) d u d v \\
& =\int_{-\infty}^{+\infty} \int_{-\infty}^{+\infty} f(u, v) \delta(x-u, y-v) d u d v
\end{aligned}
$$

If there is a linear time-invariant motion $T$, then

$$
\begin{aligned}
& T f(x, y)=\int_{-\infty}^{+\infty} \int_{-\infty}^{+\infty} f(u, v) T \delta(u-x, v-y) d u d v \\
& \text { from Definition 2.2, } \\
& T f(x, y)=(f * T \delta)(x, y) \\
& \text { taking fourier transform on both sides of }(5), \\
& \text { a } \widehat{T f}\left(\omega_{1}, \omega_{2}\right)=\widehat{f}\left(\omega_{1}, \omega_{2}\right) \widehat{T \delta}\left(\omega_{1}, \omega_{2}\right) \\
& \text { when } \widehat{T \delta}\left(\omega_{1}, \omega_{2}\right) \neq 0 \text {, we have } \\
& \widehat{f}\left(\omega_{1}, \omega_{2}\right)=\frac{\widehat{T f}\left(\omega_{1}, \omega_{2}\right)}{\widehat{T \delta}\left(\omega_{1}, \omega_{2}\right)}
\end{aligned}
$$

taking inverse fourier transform, we get

$$
\widehat{f}(x, y)=\left(\frac{\widehat{T f}\left(\omega_{1}, \omega_{2}\right)}{\widehat{T \delta}\left(\omega_{1}, \omega_{2}\right)}\right)^{\vee}(x, y)
$$

Remark: The blur effect $T$ is assumed to be linear and time-invariant and the original image is assumed to be a stationary random process. These assumptions are very 
common in dealing with practical image restoration algorithms[1, 2, 3, 4].

\section{EXPERIMENT RESULTS}

To demonstrate the performance of our method, the procedure outlined in Section 2 was implemented in experiments for commonly used standard images, all of size $256 \times 256$.

Under the known blurred parameters, we show restoration images in Fig.1, Fig.2 with blurred direction $55^{\circ}, 40^{\circ}$, the extent 10 pixels, 100 pixels, respectively.

But in the practical application, the blurred parameters are unknown and the images are disturbed by different amount of noise. Using the methods in [5], one can identify important blurred parameters. The Fig. 3 and Fig. 4 imply the restoration results of images with salt-pepper noise and gaussian noise.

For Fig.1 and Fig.2, the quality of an estimated image was measured by the conventional normalized mean square error(NMSE) and peak signal-tonoise ratio (PSNR). For Fig. 3 and Fig.4, the quality of an estimated image was measured by the conventional gray mean grads(GMG) and Laplacian(LS). The less the NMSE is, the less the resemblance is. The PSNR, GMG and LS are opposite to the NMSE. The computational results are summarized in Table 1.

\section{REFERENCES}

[1] M. Cannon, Blind deconvolution of spatially invariant image blurs with phase, IEEE Trans. Acoust. Speech Signal Process. ASSP-24, 1976.

[2] S. C. Som, Analysis of the effect of linear smear on photographic images, J. Opt. Soc. Am. 61, 1971, 859-864.

[3] A. E. Savakis and H. J. Trussell, Blur identification by residual spectral matching, IEEE Trans. Image Process. 2, 1993, 141-151.

[4] A. Rosenfeld and A. C. Kak, Digital Picture Processing, Vol. 2, Academic Press, New York, 1982.

[5] Y. Yitzhaky and N. S. Kopeika, Identification of Blur Parameters from Motion Blurred Images, GRAPHICAL MODELS AND IMAGE PROCESSING, Vol. 59, No. 5, September, pp. 310-320, 1997.

\section{[Contacts]}$$
132021
$$

Number 65 Hanyang Road Longtan District Jilin City Jilin Province Jilin Technology College of Electronic Information, Yin Jiang-yan

Tel:13500989057 E-mail:yjy197248@163.com
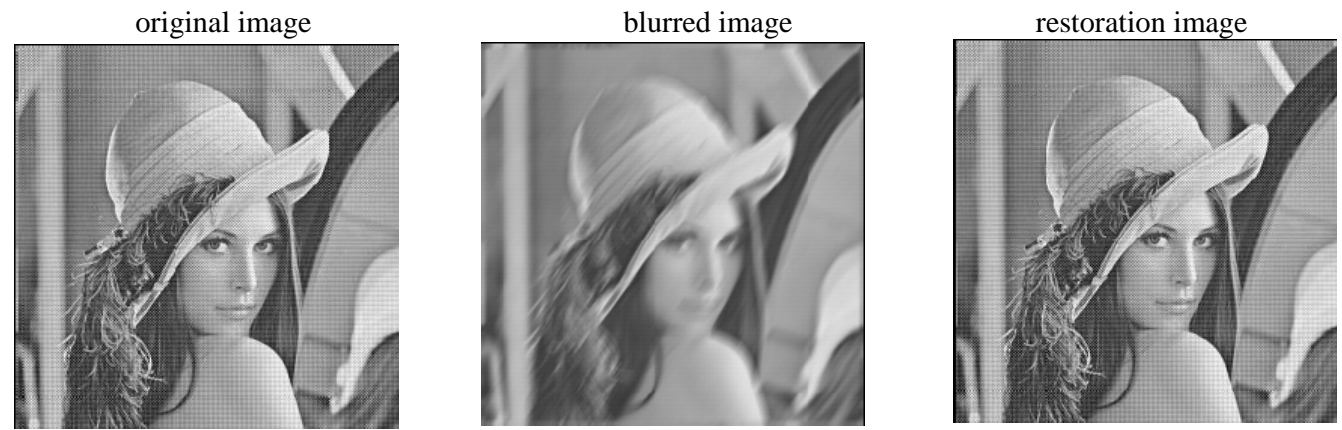

Figure 1: Blurred direction $55^{\circ}$, the extent 10 pixels
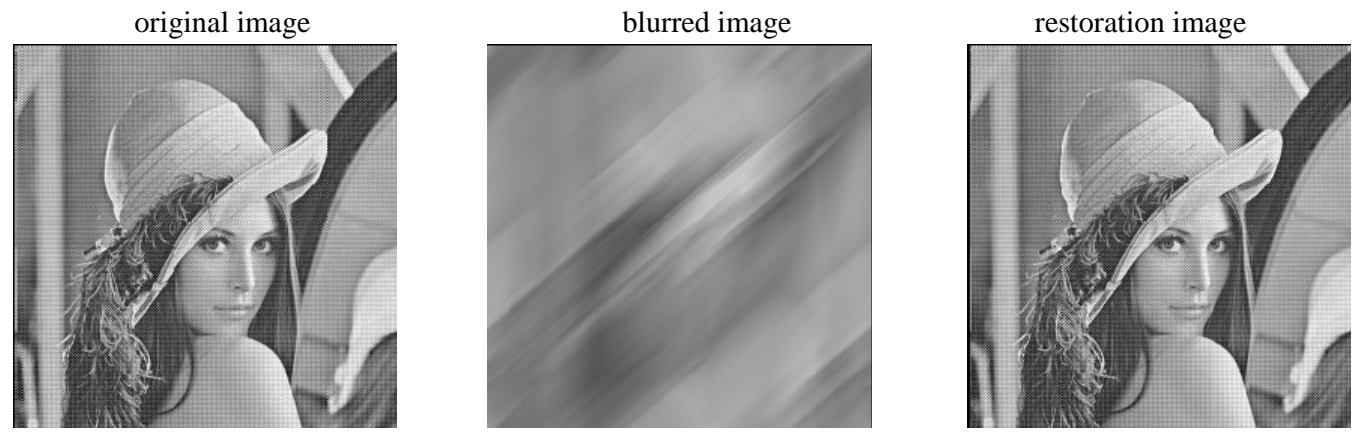

Figure 2: Blurred direction $40^{\circ}$, the extent 100 pixels 
original image

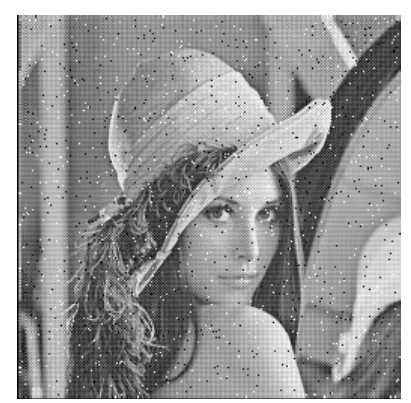

blurred image

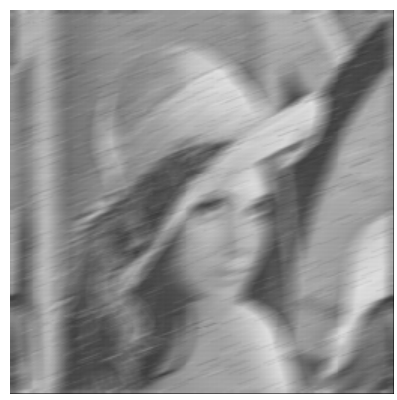

restoration image

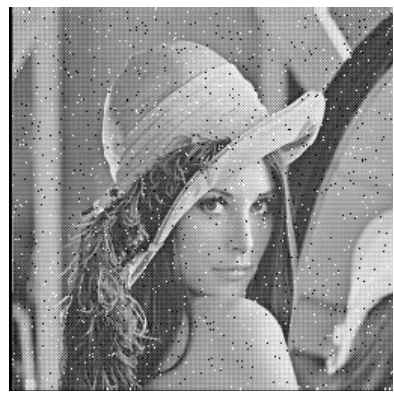

Figure 3: Blurred direction $20^{\circ}$, the extent 15 pixels with salt-pepper noise

original image

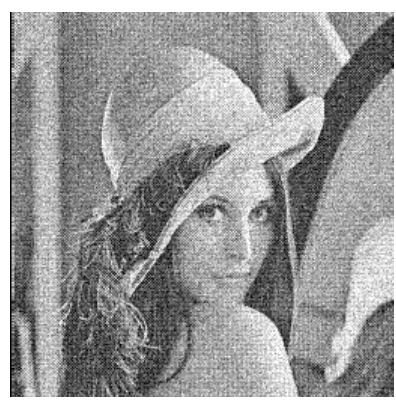

blurred image

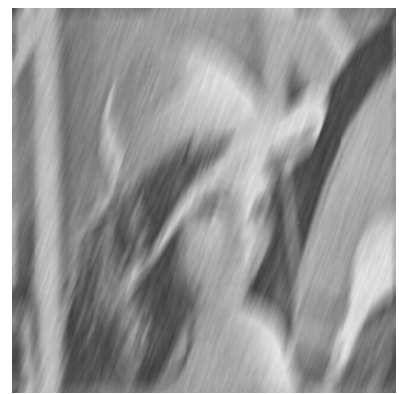

restoration image

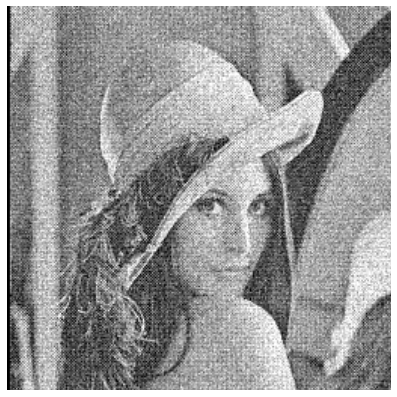

Figure 4: Blurred direction $70^{\circ}$, the extent 20 pixels with gaussian noise

TABLE I. IMAGE QUALITY ASSESSMENT

\begin{tabular}{lcrccc}
\hline & NMSE & PSNR & & GMG & LS \\
\hline Fig.1 & $6.810 \mathrm{E}-26$ & 256.310 & Fig.3 & 31.655 & 154.424 \\
Fig.2 & $2.992 \mathrm{E}-23$ & 229.882 & Fig.4 & 41.680 & 215.182 \\
\hline
\end{tabular}

